development of adhesions and membrane formation, the perinatal risk factor is recommended.

\section{IDDF2018-ABS-0238 UNCOVERED DILEMMAS REGARDING VACCINATION IN INFLAMMATORY BOWEL DISEASE - SHOULD WE FOLLOW THE WEST?}

\begin{abstract}
${ }^{1}$ Nagesh Kamat*, ${ }^{2}$ Sumit Bhatia, ${ }^{3}$ Deepti Chopra, ${ }^{4}$ Shiran Shetty, ${ }^{5}$ Anurag Shetty, ${ }^{4}$ Shabeer Ahamed. 'National Institute of Medical Statistics, Indian Council of Medical Research, New Delhi, India; ${ }^{2}$ Institute of Digestive and Hepatobiliary Sciences, Medanta The Medicity, Gurugram, Haryana, India; ${ }^{3}$ Madhukar Rainbow Children's Hospital, Malviya Nagar, New Delhi, India; ${ }^{4}$ Department of Gastroenterology and Hepatology, Kasturba Medical College, MAHE, Manipal, India; ${ }^{5}$ Department of Gastroenterology and Hepatology, Kasturba Medical College, MAHE, Mangalore, India
\end{abstract}

\subsection{6/gutjnl-2018-IDDFabstracts. 169}

Background Vaccination is the most cost-effective strategy in disease prevention but remains an ambiguous area in Inflammatory Bowel Disease (IBD). Most patients will require immunomodulators/biologics at some time point; hence there exists a large unmet need for vaccination. Guidelines are not strict around the world while western guidelines are not pragmatic in Indian population; extrapolating may not be cost-effective. However, certain diseases are uncommon in Asia-Pacific countries including India. We gathered details on the recommendation for vaccination in IBD around the world, explored the appropriateness, and calculated the probable cost of implementing them.

Methods A computer-assisted publication search of MEDLINE was performed on August 9, 2017, to identify guidelines and/ or recommendations (in English) from the year 1980-2017 for vaccination in IBD which were later critiqued. Current Index of Medical Specialties (August 2017) was used for costing, expressed in Indian Rupee (INR) and UK pound sterling $(£)$.

Results This narrative review included nine articles, all highlighting the need to assess immunosuppressed state prior to administering live vaccines, while inactivated can be used safely. Hepatitis A, Meningococcal, Japanese encephalitis and Yellow fever vaccine (for travellers) could perhaps be withheld in Indian patients with IBD since their occurrence is low. Hepatitis B, Varicella Zoster (VZ), pneumococcal, HPV and yearly influenza shots look appropriate; rest should be decided on clinical wisdom based on the type of flare (MMR, DPT if unvaccinated). Majority of the vaccines have been available after 1980, patients born prior might need additional protection. The total cost of vaccinating per patient with IBD in the Indian context is INR 48016 ( $£$ 534).

Conclusions The timing of vaccination is crucial; patients with IBD need it regardless of age. Screening is necessary, but some can be excluded, rest decided on local disease prevalence. The disease should be in remission, medication optimised and health care maintenance updated. The need for specific vaccines should currently follow national guidelines. Proposed strategy preferably would be the development of Indian guidelines for vaccination in IBD. The cost of VZ, pneumococcal and Hepatitis $\mathrm{A} / \mathrm{B}$ combination vaccine is colossally highlighting the need for prescribing cost-effective alternate vaccine brands when available.
IDDF2018-ABS-0242 SLEEVE OMENTOPEXY OVER PANCREATICO JEJUNOSTOMY - A NEW TECHNIQUE

Naseem Akhtar* . King George's Medical University, India

\subsection{6/gutjnl-2018-IDDFabstracts. 170}

Background Pancreatic anastomotic leak is considered as one of the most feared complications of Whipple's procedure. We developed a novel technique of pancreaticojejunostomy. With the use of this technique, there was no pancreatic anastomotic leak and very low rate of bleeding complications.

Methods This technique was used in 18 patients with periampullary carcinoma who underwent pancreaticoduodenectomy from December 2016 to Jan 2018. Pancreaticojejunostomy was done using standard Blumgart's technique. The anastomotic line was covered all around by a sleeve of omentum. Preoperative and intraoperative characteristics of the patients were recorded. Postoperative complications were monitored.

Results We used this technique in 18 patients. The median preoperative bilirubin and serum protein were $2.45 \mathrm{mg} / \mathrm{dl}$ and $7.1 \mathrm{gm} / \mathrm{dl}$ respectively. Ten patients had obstructive jaundice. Of which four patients had a history of preoperative CBD stenting. Median main pancreatic duct diameter was $3 \mathrm{~mm}$. The mean operating time was 5 hours and $30 \mathrm{~min}$. There was no postoperative pancreatic leak. No patient had a bleeding complication. Two patients had a hepaticojejunostomy leak, which were managed conservatively. The Median postoperative hospital stay was 16 days. There was no mortality related to the pancreatic leak.

Conclusions Sleeve omentopexy is a very simple technique without any added cost. This procedure hardly adds to the operative time. Till now this technique has markedly reduced pancreatic leak and bleeding-related complications in our department. As we grow our experience with this technique in more and more patients, we can come up with some robust data.

\section{IDDF2018-ABS-0246 MUCOSAL HEALING REDUCED THE OCCURRENCE AND RECURRENCE OF CLINICAL DISABILITY IN CROHN'S DISEASE}

Shanshan Huang*, Shenghong Zhang, Minhu Chen. Department of Gastroenterology, The First Affiliated Hospital, Sun Yat-sen University, China

\subsection{6/gutjnl-2018-IDDFabstracts. 171}

Background Mucosal healing ( $\mathrm{MH})$ is the 'target' of 'treat to target' strategy in Crohn's disease (CD), which seeks to prevent disability. However, data is limited on whether achieving $\mathrm{MH}$ could reduce disability in $\mathrm{CD}$. We aimed to evaluate the probability of disabling $\mathrm{CD}$ and explore the effect of $\mathrm{MH}$ achievement on clinical disability in CD.

Methods This was a retrospective cohort study of 319 consecutive $\mathrm{CD}$ patients in a tertiary hospital in China. $\mathrm{MH}$ was defined as the absence of ulcerations. The primary outcome was a clinical disability, which was defined as one of the followings:

1. more than one surgery [index surgery excluded];

2. new events such as penetrating, perianal disease or stricture;

3. at least three hospitalizations;

4. switching therapy;

5. adverse steroid events. 